\title{
A Comparison of Fatigue, Level of Physical Activity, and Sleep Quality in Pregnant and Non-Pregnant Nurses Working 16-Hour Night Shifts in
} Japan

\author{
Narumi Ooshige ${ }^{*}$, , Tae Yamaguchi ${ }^{1}$, Mitsuyo Nakashima ${ }^{2}$, Ryohei Kimura $^{3}$, Takuro Tobina ${ }^{1}$, Miyuki Nagamatsu ${ }^{3}$ and \\ Yumiko Kuraoka ${ }^{3}$
}

${ }^{1}$ Department of Nursing, Faculty of Nursing and Nutrition, University of Nagasaki, Nagasaki 851-2195, Japan

${ }^{2}$ Department of Nursing, School of Medicine, University of Fukuoka, Fukuoka 814-0180, Japan

${ }^{3}$ Department of Nursing, Japanese Red Cross Kyushu International College of Nursing, Fukuoka 811-4157, Japan

\section{Abstract}

Background: Most medical institutions require long work hours, making nurse health risk an important issue.

Methods: This longitudinal study involved 26 pregnant and non-pregnant Japanese nurses in whom we compared fatigue, intensity of physical activity, and quality of sleep for each working time throughout the course of the day shift, night shift, and day off.

Results: Subjective fatigue increased after each shift. The cumulative fatigue symptoms index (CFSI) score was higher in non-pregnant nurses. Continued sympathetic nerve predominant state and higher activity level were found in non-pregnant nurses compared to pregnant nurses.

Conclusion: Non-pregnant nurses had a higher level of fatigue according to CFSI, and they were in a stressful state throughout the night shift.

\section{Background}

A large-scale cohort study of female nurses engaged in shift work revealed that continuously working night shifts increases the risk of coronary heart disease (CHD). Furthermore, it was found that the risk of CHD reduces after a long period has passed after quitting such shift work [1]. Moreover, it has also been reported that based on the characteristics of shift work, correlations with fatigue are observed following night shifts and while awaiting leave [2].

According to a survey of Japanese nurses, a two-shift system accounts for approximately $30 \%$ overall, and $43.1 \%$ nurses work prolonged night shifts of $\geq 16$ hours [3]. The proportion of prolonged night shifts of $\geq 16$ hours has not changed in recent years and accounts for over half at $52.5 \%$, which is a serious situation eliciting concern over the impact of long night shifts on patient safety and the health of nursing staff.3When subjective fatigue persists, it leads to cumulative fatigue, which makes it difficult to recover. Clinical experience $<5$ years, subjective busyness in night shift work, and the number of steps on a day off as a measure of physical activity were identified as factors associated with Cumulative Fatigue Symptoms Index (CFSI) in nurses working in two-shift systems [4]. However, no studies related to cumulative fatigue in pregnant nurses are available. Furthermore, in hospitals with a heavy night shift load, the turnover rate tends to be high, and the most common reason for the nursing staff to quit their jobs is for "childbirth and childcare," accounting for $22.1 \%$ [5]

No universal coping method has been established for shift workers who need to change their sleeping time zone intentionally. A previous study of sleep among pregnant women revealed that as a change associated with the progression of pregnancy, nocturnal awakening is common in the first trimester. As pregnancy progresses into the third trimester, the Pittsburgh Sleep Quality Index (PSQI) score increases, indicating poorer sleep quality [6]. In Japan, it has been reported that sleep is disturbed more at one month postpartum than in the first and third trimesters, whereas the sense of difficulty in sleeping was better postpartum than in the second and third trimesters [7]. However, the subjects of these reports were all women of the general population. Physical inactivity has garnered attention as a factor that affects sleep disturbance [8]. Moreover, it has been reported that physical activity can hinder fatigue [9]. Therefore, it has been found that sleeping status is related to the level of physical activity and fatigue. However, the actual situation for nurses who work 16-hour night shifts in a twoshift system comparing pregnant and non-pregnant nurses is unclear. Therefore, we thought that clarifying the characteristics of each period by comparing fatigue, physical activity intensity, and quality of sleep according to pregnant and non-pregnant nurses would provide support to enable continued employment while maintaining performance.

We aimed to elucidate the differences between pregnant and nonpregnant nurses in their 20s through 30s working 16-hour night shifts in a two-shift system using subjective and objective indicators of fatigue, physical activity intensity, and quality of sleep throughout the flow of a day shift, night shift, and day off.

\section{Materials and Methods}

\section{Participants}

The subject sample consisted of 26 nurses working 16-hour night shifts in a two-shift system and who were employed at four general hospitals with at least 160 beds and patient-nurse ratio of 7:1. The nurses were in their 20 s through 30 s with no underlying diseases, such as sleeping disorders, and the number of years of nursing experience

"Corresponding Author: Prof. Narumi Ooshige, Faculty of Nursing and Nutrition, Department of Nursing, University of Nagasaki, 1-1-1 Manabino Nisisonogi-gun, Nagasaki 851-2195, Japan, Tel/Fax: +81-958-13-5175; E-mail: n-ooshige@sun.ac.jp

Citation: Ooshige N, Yamaguchi T, Nakashima M, Kimura R, Tobina T, et al. (2021) A Comparison of Fatigue, Level of Physical Activity, and Sleep Quality in Pregnant and Non-Pregnant Nurses Working 16-Hour Night Shifts in Japan. Int J Nurs Clin Pract 8: 344. doi: https://doi.org/10.15344/2394-4978/2021/344

Copyright: (C) 2021 Ooshige et al. This is an open-access article distributed under the terms of the Creative Commons Attribution License, which permits unrestricted use, distribution, and reproduction in any medium, provided the original author and source are credited. 
Citation: Ooshige N, Yamaguchi T, Nakashima M, Kimura R, Tobina T, et al. (2021) A Comparison of Fatigue, Level of Physical Activity, and Sleep Quality in Pregnant and Non-Pregnant Nurses Working 16-Hour Night Shifts in Japan. Int J Nurs Clin Pract 8: 344. doi: https://doi.org/10.15344/2394-4978/2021/344

was not taken into consideration. The survey period was from August 2019 to December 2020.

The shift schedule of the participating institutions included a day shift from 8:30 to 17:00 and night shift from 16:30 to 9:00 at three out of four hospitals. In the remaining hospital, the day shift was from $8: 30$ to $17: 30$, and the night shift was from $16: 15$ to $9: 15$. Accordingly, in all subjects, we surveyed a consecutive four-day period involving a day shift (8 hours), night shift (16 hours), and day off after night shift.

\section{Data collection}

We used the questionnaire "Jikaku-sho shirabe" as a subjective scale of fatigue [10] and CFSI. For measuring the intensity of physical activity, we used the metabolic equivalent of tasks (METs). To measure the quality of sleep, we used the Japanese version of the PSQI (PSQI-J) [11]. As an objective measure of sleep, we used high frequency components (HF), low frequency (LF)/HF, and heart rate (HR) $[12,13]$.

\section{Fatigue}

\section{Subjective fatigue called Jikaku-sho Shirabe}

The Jikaku-sho shirabe was measured a total of six times before and after the day shift, before and after the night shift, and before and after the day off. This scale had confirmed construct validity. The questionnaire consisted of a total of 25 question items divided into five groups. Subjective complaints of fatigue symptoms were classified into five groups, consisting of five items each, as follows: group I drowsiness, group II: feeling of instability, group III: uneasiness, group IV: tiredness, and group V: eyestrain. Thus, fatigue can be perceived from multiple angles. Scores for each group ranged from 5 to 25 points, with higher scores indicating greater subjective fatigue [10]

\section{CSFI}

As a scale to evaluate cumulative fatigue, we used the CFSI. CFSI consists of eight characteristic items from three aspects: mental aspect items of "depressive feelings," "anxiety," and "decreased vitality;" physical aspect items of "general fatigue," "chronic tiredness," and "physical disorders;" and social aspect items of "decreased willingness to work" and "irritability." Responses to the 81 question items were provided according to two choices, i.e., "yes" or "no," and compared as a mean rate of complaint for each scale [14]

\section{HF, LF/HF, and HR}

Using a WHS- $1^{\oplus}$ wearable heart beat sensor from Union Tool Co., we measured subject HR during sleep after the day shift and when napping during the night shift. The frequency of $0.04-0.40 \mathrm{~Hz}$ in $\mathrm{HR}$ variability of the autonomic nervous system was included; the high frequency component $(>0.15 \mathrm{~Hz})(\mathrm{HF})$, low frequency component (0.04-0.15 Hz) (LF), and LF-to-HF ratio (LF/HF) were each extracted every 5 minutes. The mean values were then calculated. The HF and $\mathrm{LF} / \mathrm{HF}$ were used as indices of parasympathetic nerve activity and sympathetic nerve activity, respectively, and reflected fatigue and stress $[15,16]$. The $\mathrm{LF} / \mathrm{HF}$ reference value was $<2.0$.

\section{Physical activity}

Subjects wore a Lifecorder GS ${ }^{\star}$ from Suzuken Co. from prior to the start of the day shift throughout the night shift and day off, except when taking a bath, using which physical activity was measured by sampling the flow of the physical activity level every $4 \mathrm{~s}$ and converting it into 2-minute periods. The METs of each shift were classified into four types, which included sedentary behavior (SB) at resting level, light physical activity (LPA) at walking level, moderate physical activity (MPA) at brisk walking level, and vigorous physical activity (VPA) at jogging level. The numerical classification of METs was SB: $<1.5$ METS, LPA: $\geq 1.5$ METS and $<3.0$ METs, MPA: $\geq 3.0$ METS and $<6.0$ METs, and VPA: $\geq 6.0$ METs $[17,18]$. The measurement times were 08:00-18:00 in the day shift, 16:00-09:00 in the night shift, and 0:00-24:00 on the day off.

\section{PSQI-J}

The PSQI-J is widely used for the evaluation of sleep disturbance, with which seven factors including quality of sleep, time to fall asleep, sleep duration, sleep efficiency, sleep difficulty, use of sleeping pills, and difficulty waking up in the day are calculated to give a total score. Total scores of the PSQI-J range from 0-21 points, with higher scores judged to indicate greater sleep disturbance [11].

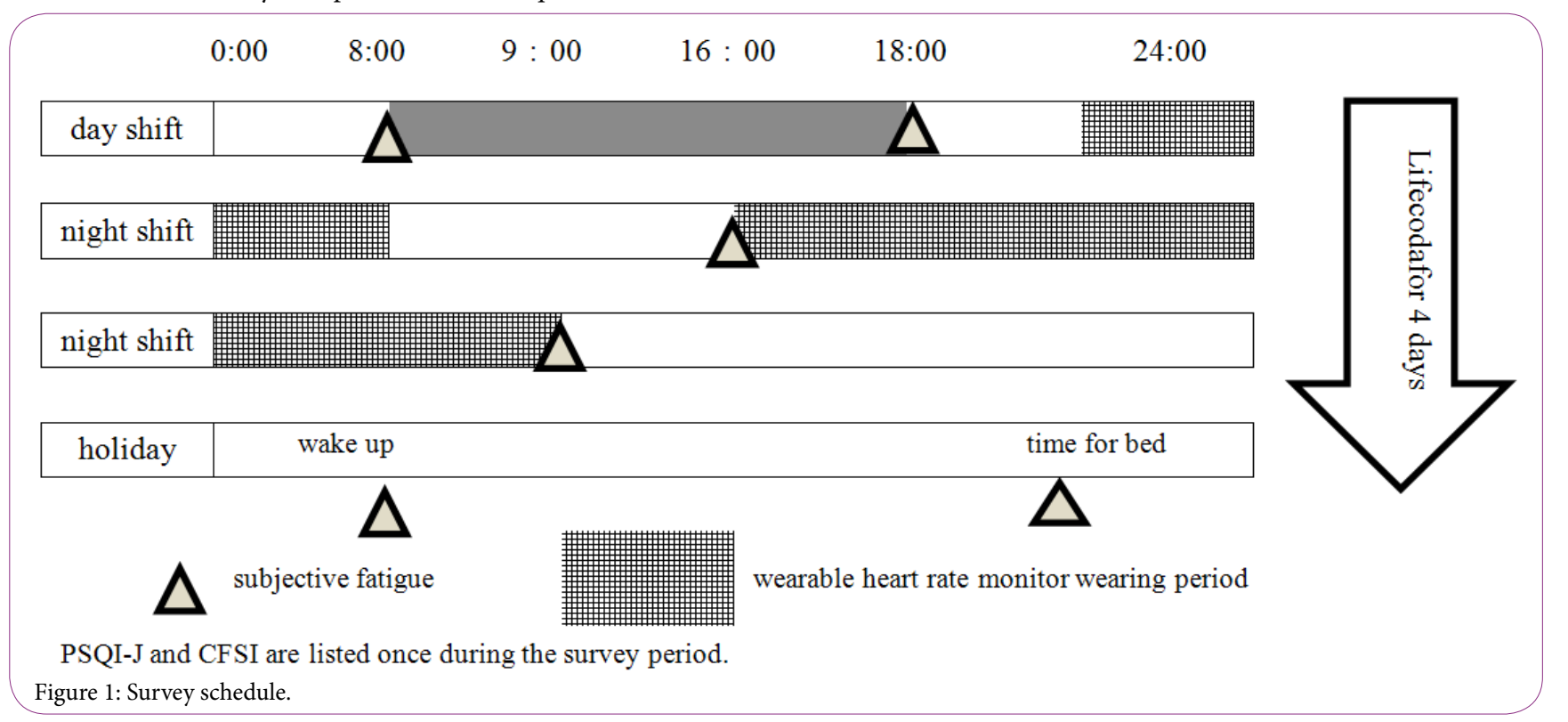


Citation: Ooshige N, Yamaguchi T, Nakashima M, Kimura R, Tobina T, et al. (2021) A Comparison of Fatigue, Level of Physical Activity, and Sleep Quality in Pregnant and Non-Pregnant Nurses Working 16-Hour Night Shifts in Japan. Int J Nurs Clin Pract 8: 344. doi: https://doi.org/10.15344/2394-4978/2021/344

Page 3 of 8

\section{Survey schedule}

Jikaku-sho Shirabe was measured before and after work, and a WHS $-1^{\circ}$ wearable heart beat was used and compared during nighttime sleep and during night shift. PSQI-J and CFSI are listed once during the survey period (Figure 1).

\section{Ethical approval and informed consent}

Ethical considerations were implemented with the approval of the Research Ethical Review Board of the Japanese Red Cross Kyushu International College of Nursing, with which the researcher is affiliated (Approval Number: 19-005-(1)).

\section{Results}

\section{Participant characteristics}

Pregnant nurses had an average pregnancy week of 23.5 weeks (SD 5.2 weeks).The unpaired t-test used that the mean age was significantly higher for the non-pregnant nurses when compared with the pregnant nurses $(\mathrm{p}<0.05)$. Besides, although no significant difference was noted, the number of steps in each shift was higher for the non-pregnant nurses than for the pregnant nurses. Fisher's exact test used that most pregnant nurses cohabited with their family $(\mathrm{p}<$ 0.05) (Table 1).

\begin{tabular}{|c|c|c|c|c|c|}
\hline Variable & & $\mathrm{n}$ & Mean & SD & $p$-value \\
\hline \multirow[t]{2}{*}{ Age } & Pregnant & 11 & 29.5 & 3.8 & $0.029^{\mathrm{a}}$ \\
\hline & Non- Pregnant & 15 & 34.1 & 5.6 & \\
\hline \multirow[t]{2}{*}{ Years of experience } & Pregnant & 11 & 7.2 & 4.0 & $0.081^{\mathrm{a}}$ \\
\hline & Non- Pregnant & 15 & 10.6 & 5.2 & \\
\hline \multirow[t]{2}{*}{ Numbers of month of night shift experience } & Pregnant & 11 & 75.9 & 48.2 & $0.118^{\mathrm{a}}$ \\
\hline & Non- Pregnant & 15 & 114.7 & 67.7 & \\
\hline \multirow[t]{2}{*}{ Numbers of night shifts/months } & Pregnant & 11 & 4.5 & 1.6 & $0.326^{\mathrm{a}}$ \\
\hline & Non- Pregnant & 15 & 5.1 & 1.4 & \\
\hline \multirow[t]{2}{*}{ Bed time after day shifts } & Pregnant & 11 & $22: 06$ & 40 & $0.545^{\mathrm{a}}$ \\
\hline & Non- Pregnant & 15 & 23:05 & 55 & \\
\hline \multirow[t]{2}{*}{ Wake-up time after night shifts } & Pregnant & 11 & $6: 33$ & 1.53 & $0.361^{\mathrm{a}}$ \\
\hline & Non- Pregnant & 15 & 7:05 & 1.01 & \\
\hline \multirow[t]{2}{*}{ Nap start time during night shifts } & Pregnant & 11 & $1: 40$ & 2.24 & $0.793^{\mathrm{a}}$ \\
\hline & Non- Pregnant & 15 & $1: 53$ & 1.44 & \\
\hline \multirow[t]{2}{*}{ End time of nap during night shifts } & Pregnant & 11 & $3: 10$ & 2.13 & $0.432^{\mathrm{a}}$ \\
\hline & Non- Pregnant & 14 & $3: 48$ & 1.42 & \\
\hline \multirow[t]{2}{*}{ Nap time } & Pregnant & 11 & 109 & 20 & $0.246^{\mathrm{a}}$ \\
\hline & Non- Pregnant & 15 & 100 & 19 & \\
\hline \multirow[t]{2}{*}{ PSQI-J } & Pregnant & 11 & 6.9 & 2.8 & $0.664^{\mathrm{a}}$ \\
\hline & Non- Pregnant & 15 & 7.5 & 4.0 & \\
\hline \multirow[t]{2}{*}{ Daily shift steps } & Pregnant & 11 & 9635 & 2128 & $0.093^{\mathrm{a}}$ \\
\hline & Non- Pregnant & 15 & 12770 & 5638 & \\
\hline \multirow[t]{2}{*}{ Night shift steps } & Pregnant & 11 & 16334 & 3703 & $0.223^{\mathrm{a}}$ \\
\hline & Non- Pregnant & 15 & 22047 & 14734 & \\
\hline \multirow[t]{4}{*}{ Day of steps } & Pregnant & 11 & 4707 & 2771 & $0.122^{\mathrm{a}}$ \\
\hline & Non- Pregnant & 15 & 8069 & 6475 & \\
\hline & \multicolumn{2}{|c|}{ Pregnant $(\mathrm{n}=11)$} & \multicolumn{2}{|c|}{ Non- Pregnant $(n=15)$} & \multirow[t]{2}{*}{$P$-value } \\
\hline & $\mathrm{n}$ & $\%$ & $\mathrm{n}$ & $\%$ & \\
\hline Prenatal & 8 & 53.3 & 7 & 46.7 & \multirow[b]{2}{*}{$0.184^{\mathrm{b}}$} \\
\hline Multipara & 3 & 27.3 & 8 & 72.7 & \\
\hline Family living together & 11 & 52.4 & 10 & 47.6 & \multirow[b]{2}{*}{$0.033^{b}$} \\
\hline Non-family living together & 0 & 0 & 5 & 100.0 & \\
\hline Children living together & 3 & 23.1 & 7 & 72.7 & \multirow[b]{2}{*}{$0.315^{\mathrm{b}}$} \\
\hline Non-Children living together & 8 & 61.5 & 8 & 46.7 & \\
\hline $\begin{array}{l}\text { Table 1: Chracteristics of the participants. } \\
\text { unpaired t test } \\
\text { Fisher's exact test }\end{array}$ & & & & & \\
\hline
\end{tabular}


Citation: Ooshige N, Yamaguchi T, Nakashima M, Kimura R, Tobina T, et al. (2021) A Comparison of Fatigue, Level of Physical Activity, and Sleep Quality in Pregnant and Non-Pregnant Nurses Working 16-Hour Night Shifts in Japan. Int J Nurs Clin Pract 8: 344. doi: https://doi.org/10.15344/2394-4978/2021/344

Page 4 of 8

\section{Jikaku-sho Shirabe and CFSI scores}

The two-way analysis of variance results revealed a significant difference in the intra-subject test of all Jikaku-sho shirabe symptoms, excluding the feeling of instability $(\mathrm{p}<0.01)$; however, there was no significant difference between the subjects. Furthermore, no interaction was observed for any of the symptoms (Figure 2). In the
CFSI, higher values were found for the physical ailments of pregnant women compared to general female Japanese laborers $(n=23,835)$ [14]; however, mental ailments had lower values. As a result of the multivariate analysis of variance according to the presence or absence of pregnancy, non-pregnant women more often complained of feeling depressed, experiencing poor physical health, and anxiety in the CFSI $(\mathrm{p}<0.05)$ (Figure 3).

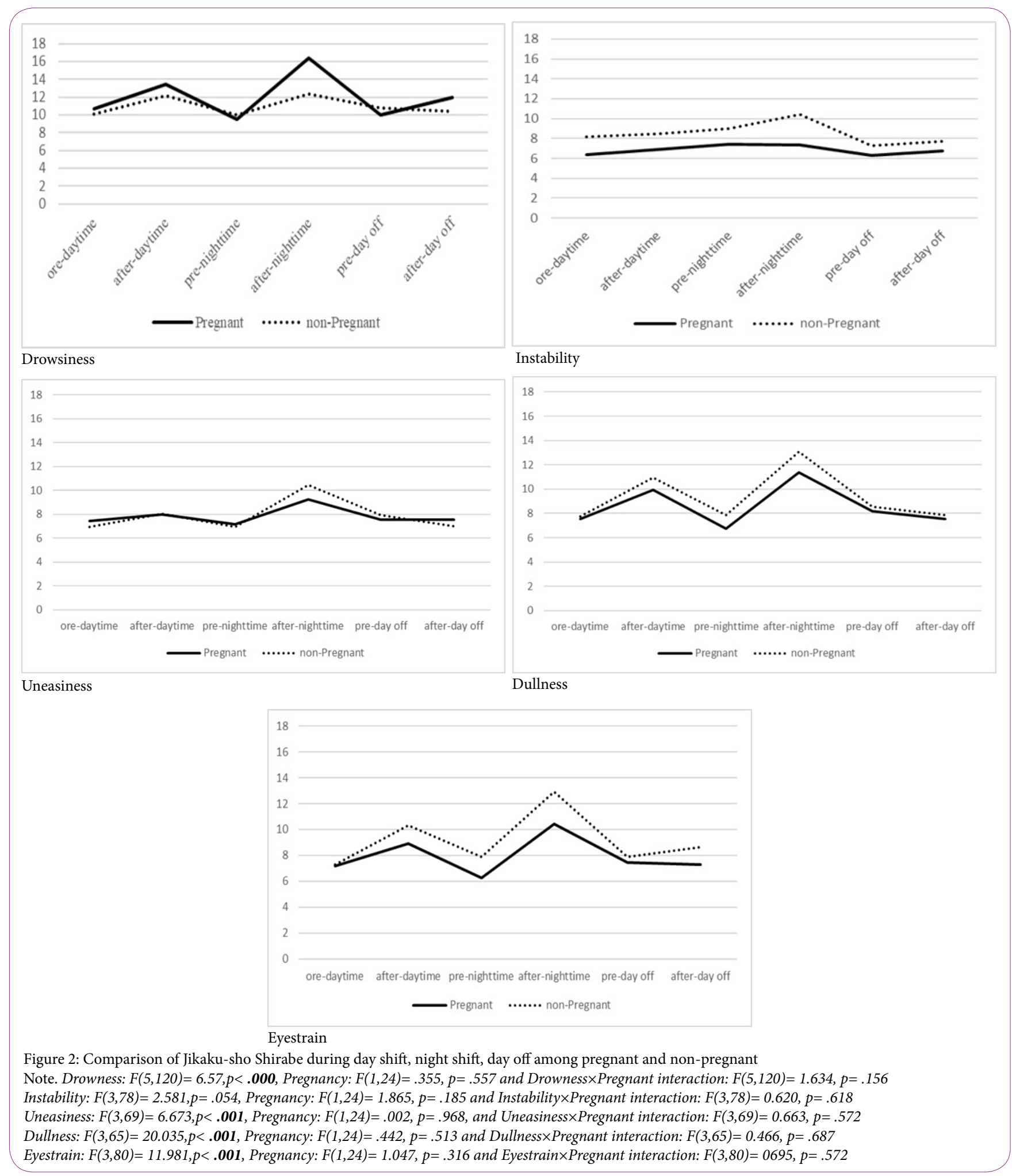


Citation: Ooshige N, Yamaguchi T, Nakashima M, Kimura R, Tobina T, et al. (2021) A Comparison of Fatigue, Level of Physical Activity, and Sleep Quality in Pregnant and Non-Pregnant Nurses Working 16-Hour Night Shifts in Japan. Int J Nurs Clin Pract 8: 344. doi: https://doi.org/10.15344/2394-4978/2021/344

Page 5 of 8

\section{HF, LF/HF, and HR}

HF and LF/HF while sleeping at night after the day shift were significantly higher in non-pregnant women, and only HR was significantly lower $(p<0.05)$. The LF/HF during the night shift was significantly higher for non-pregnant nurses $(\mathrm{p}<0.05)$. There was no significant difference observed during night shifts and when napping during night shifts. The LF/HF of non-pregnant nurses was higher than the reference value at all shift times. A robust unpaired t-test was used, even if it was not a perfect normal distribution (Table 2).

\section{Physical activity}

The SB, LPA, MPA, and VPA were compared to non-Pregnant and Pregnant in day and night shit subjects. As a result of the unpaired $\mathrm{t}$-test, LPA of the day shift was more common for pregnant nurses ( $\mathrm{p}<$ 0.05 ), while the activity of non-pregnant nurses was commonly VPA $(\mathrm{p}<0.01)$. In the night shifts, VPA was only common among the nonpregnant nurses $(\mathrm{p}<0.01)$. On the day off, no significant difference was observed (Figure 4).

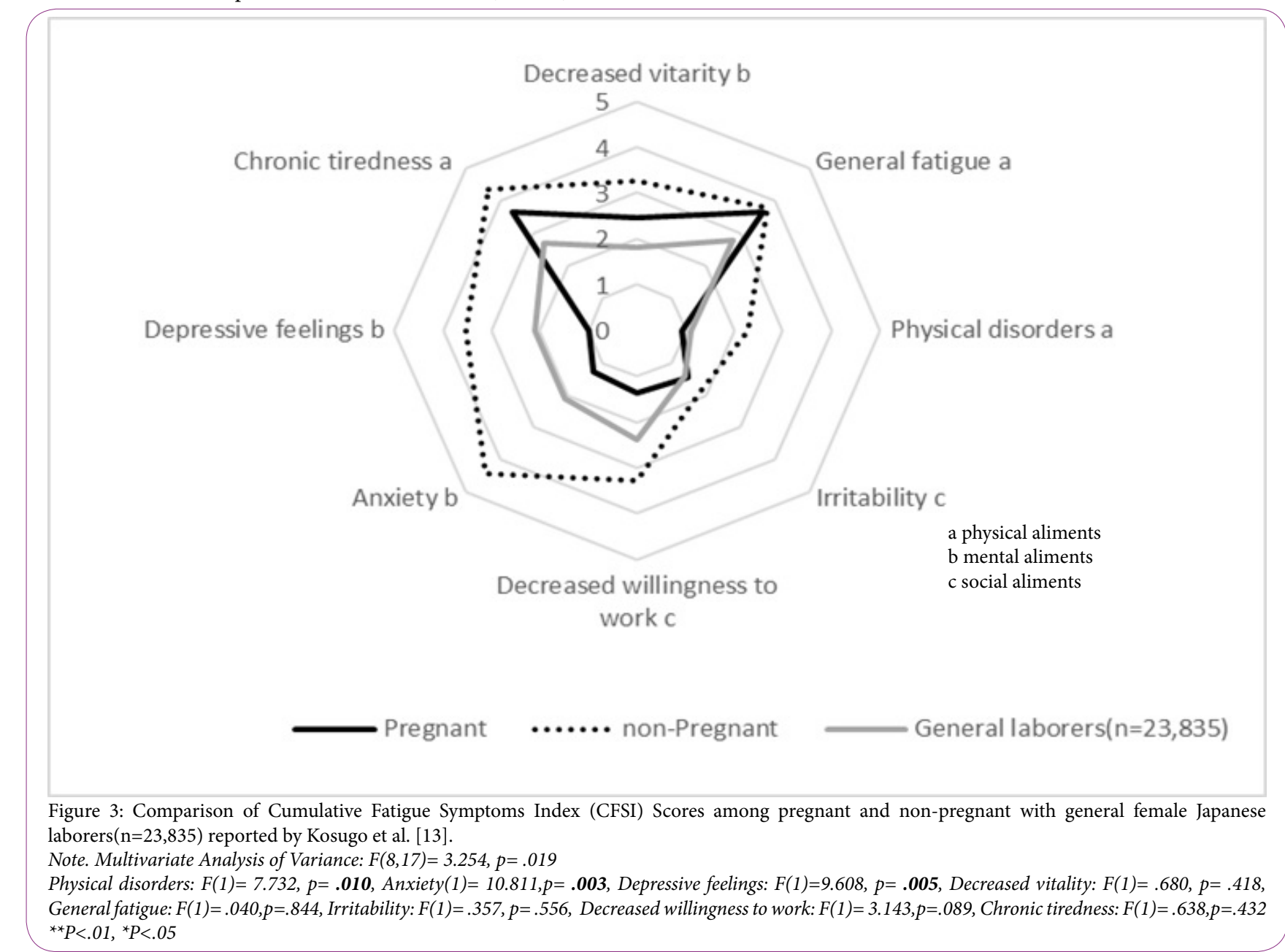

work c

b mental aliments

c social aliments

\begin{tabular}{|c|c|c|c|c|c|}
\hline & \multicolumn{2}{|c|}{ Pregnant $(\mathrm{n}=11)$} & \multicolumn{2}{|c|}{ Non-pregnant $(\mathrm{n}=15)$} & \multirow[t]{2}{*}{$p$-value } \\
\hline & Mean & SD & Mean & SD & \\
\hline Sleeping HF after day shift & 724.8 & 262.6 & 1349.3 & 1046.3 & 0.041 \\
\hline Sleeping LF/HF after day shift & 1.5 & 1.0 & 16.5 & 25.4 & 0.039 \\
\hline Sleeping HR after day shift & 72.9 & 9.4 & 63.9 & 9.1 & 0.022 \\
\hline HF during night shift & 495.1 & 190.3 & 942.9 & 845.1 & 0.065 \\
\hline LF/HF during night shift & 2.8 & 2.8 & 16.1 & 18.9 & 0.017 \\
\hline HR during night shift & 92.6 & 13.2 & 87.6 & 6.9 & 0.220 \\
\hline Night shift nap HF & 503.2 & 197.9 & 806.1 & 751.9 & 0.208 \\
\hline Night shift nap LF/HF & 2.0 & 2.6 & 56.5 & 140.3 & 0.169 \\
\hline Night shift nap HR & 82.7 & 15.9 & 71.7 & 7.7 & 0.053 \\
\hline
\end{tabular}

Table 2: Comparison of HF, LF/HF, HR among Pregnant and non-Pregnant.

unpaired t test. 
Citation: Ooshige N, Yamaguchi T, Nakashima M, Kimura R, Tobina T, et al. (2021) A Comparison of Fatigue, Level of Physical Activity, and Sleep Quality in Pregnant and Non-Pregnant Nurses Working 16-Hour Night Shifts in Japan. Int J Nurs Clin Pract 8: 344. doi: https://doi.org/10.15344/2394-4978/2021/344

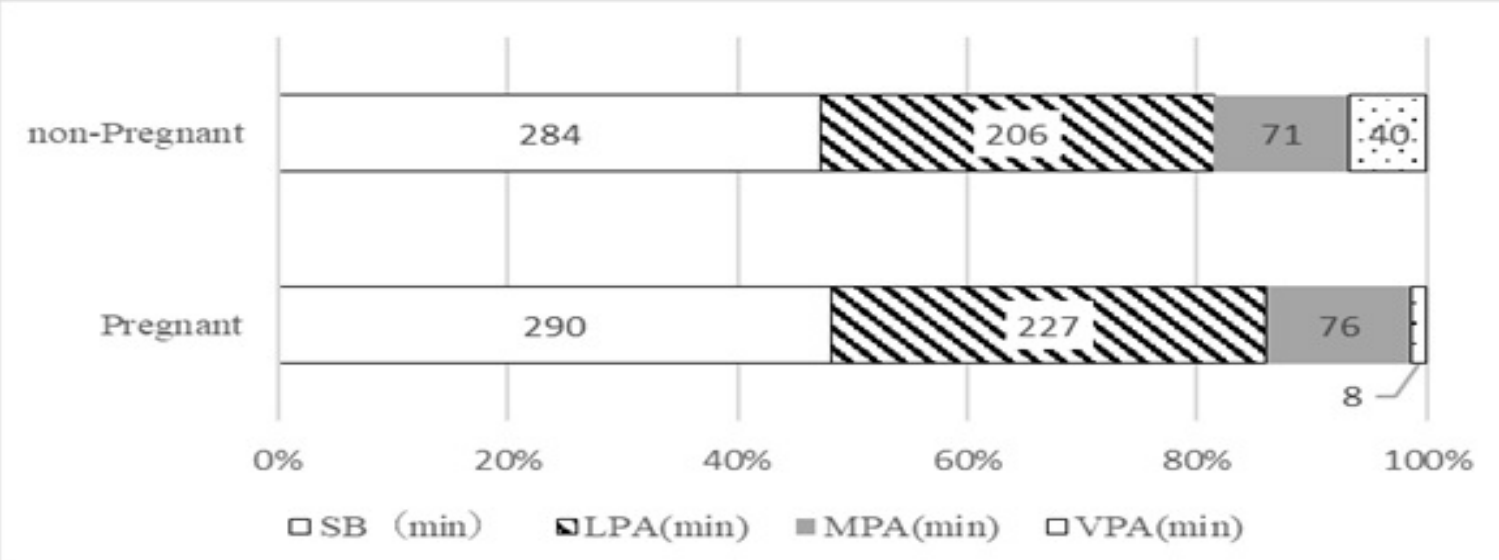

Day Shift

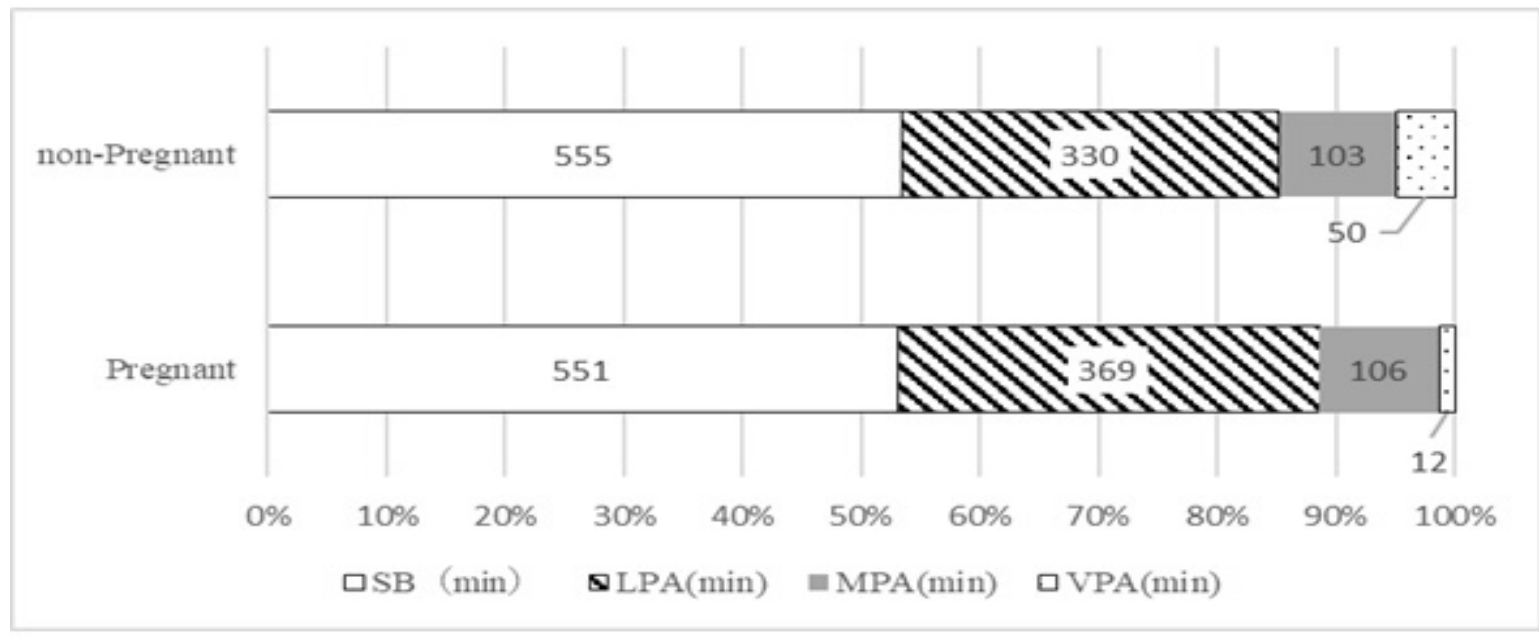

Night Shift

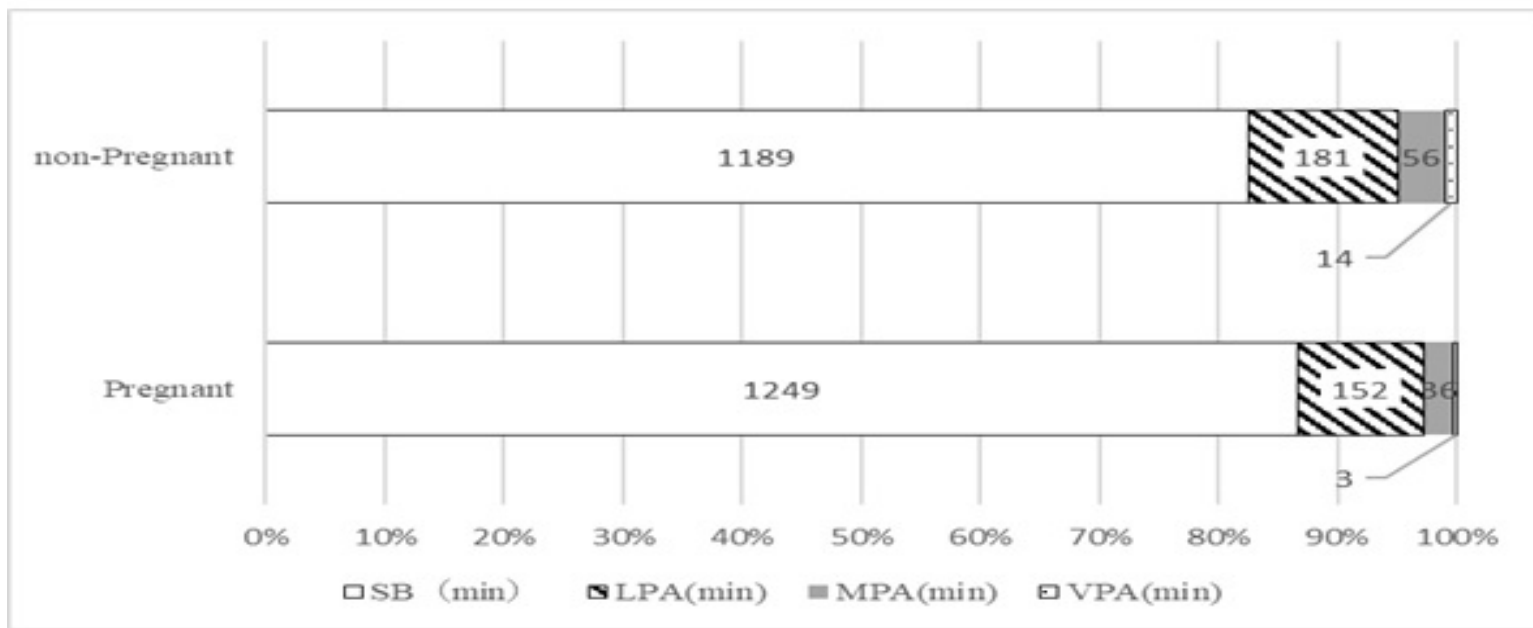

Day off

Figure 4: Comparison of Physical activity day shift, night shift, day off among pregnancy and non-pregnancy.

Note. Day shift of SB:t=.311,df=24,p=.758, Day shift of $\mathrm{LPA}: \mathrm{t}=2.117, \mathrm{df}=24, \mathrm{p}=.045$,Day shift of MPA:t=.441,df=24,p=.663, Day shift of VPA: $\mathrm{t}==^{\prime}-4.956, \mathrm{df}=17, \mathrm{p}<.001$

Night shift of SB:t='-0971,df=24,p=.923, Night shift of LPA:t=1.285,df=24,p=.211,Night shift of MPA:t=.166,df=24,p=.869, Night shift of VPA: $t={ }^{\prime}-3.415, \mathrm{df}=17, \mathrm{p}=.003$

Day off of $\mathrm{SB}: \mathrm{t}=1.609, \mathrm{df}=23, \mathrm{p}=.121$, Holiday of $\mathrm{LPA}: \mathrm{t}=\mathbf{\prime}^{\prime}-1.057, \mathrm{df}=23, \mathrm{p}=.302$, Holiday shift of MPA:t='-1.426,df=23,p=.167, Holiday of VPA:t='-2.035, $\mathrm{df}=14, \mathrm{p}<.061$ 
Citation: Ooshige N, Yamaguchi T, Nakashima M, Kimura R, Tobina T, et al. (2021) A Comparison of Fatigue, Level of Physical Activity, and Sleep Quality in Pregnant and Non-Pregnant Nurses Working 16-Hour Night Shifts in Japan. Int J Nurs Clin Pract 8: 344. doi: https://doi.org/10.15344/2394-4978/2021/344

\section{Discussion}

In the present study, the pregnant nurses had a mean age of 29 years, were mid-career nurses with 7 years of nursing experience, and had a moderately high mean PSQI-J score of 6.9; however, they did not exhibit severe sleep disturbance. The non-pregnant nurses had a mean age of 34 years. Despite being older than the pregnant women, they were similarly mid-career nurses with 10 years of experience. Furthermore, the mean PSQI-J score was 7.5 points, which was a somewhat higher score than that of the pregnant nurses, indicating worse quality of sleep, and they were more active with a greater number of steps in all shift times compared to the pregnant nurses. Moreover, as many of the non-pregnant nurses often cohabited with children, it is inferred that time taken for childcare might have led to poor quality of sleep [19].

Subjective fatigue tended to increase after day shifts; nonetheless, with regard to before and after night shifts, subjective fatigue increased after night shifts, and only the feeling of instability was unchanged from before to after the shifts. Although no significant difference was observed according to the presence or absence of pregnancy, sleepiness was more strongly felt by pregnant nurses after their night shift than by non-pregnant nurses. Increased sleepiness is a factor that easily affects the deterioration of sleep quality for pregnant women [20]. Furthermore, as sleepiness easily hinders one's duties [21], early recovery from increased sleepiness after shift completion is important.

The CFSI revealed that non-pregnant nurses complained more of mental ailments, including a feeling of depression and instability, and poor physical health, and it was inferred that the mental ailments persisted. Furthermore, the CSFI is an evaluation tool to investigate the "load phenomenon" in the work environment [22]. In a previous study of caregivers using the CFSI, it was reported that caregivers strongly complained of physical ailments, including general fatigue and chronic tiredness, and that individuals with good diet and exercise habits had a lower sense of fatigue [23]. Psychological job demands were significantly associated with acute and chronic fatigue and intershift recovery. Rotating shifts were significantly related to acute fatigue [24]. Unlike the results of previous studies [23,24], non-pregnant nurses of the present study had markedly high values for mental ailments, while pregnant and non-pregnant nurses had high values for physical ailment. This result suggests that nurses working 16-Hour Night Shifts in a two-shift system tend to have high cumulative fatigue, and in particular, non-pregnant nurses had a higher level of activity during their duties, which has a major impact on their workload.

The autonomic nervous system showed higher values for HF and $\mathrm{LF} / \mathrm{HF}$ in non-pregnant nurses than in pregnant nurses while sleeping at night after their day shift. Pregnant nurses had higher HR than non-pregnant nurses during sleep and night shifts after day shift. In particular, compared to a previous study [12] in which an LF/HF of $\geq 4$ was considered an excited state, non-pregnant nurses exhibited high values. Therefore, it was inferred that they were in a sympathetic nerve predominant stressful state even when sleeping at night on workdays. Furthermore, during night shifts and when napping during night shifts, the fact that non-pregnant nurses showed high values indicated that the stressful state persisted while napping during night shifts.

We found that subjective fatigue, except for the feeling of instability, in nurses working a 16-hour shift in a two-shift system intensified during day shifts but could be recovered by getting adequate sleep at night. Furthermore, even in night shifts, the sense of fatigue increased but recovered through the day off. This tendency was found to be the same between pregnant and non-pregnant nurses. Moreover, the autonomic nervous system results when sleeping after the day shift and during night shifts suggested that the sympathetic nerve predominant stressful state in non-pregnant nurses continued from the day shift until the night shift.

With regard to the intensity of physical activity, upon comparing the intensity of physical activity in the day shift, LPA was common in pregnant nurses and VPA was common in non-pregnant nurses. This finding implied that non-pregnant nurses carried out duties with a higher intensity of physical activity in the day shift. Comparison of pregnant nurses and non-pregnant nurses in the night shift indicated that VPA was common in non-pregnant nurses, which indicated that the non-pregnant nurses had a greater amount of work than the pregnant nurses also in the night shift continuing from the day shift. On the day off, there was no difference according to the presence or absence of pregnancy, and the intensity of physical activity was the lowest, with SB accounting for $70 \%$ of one day for both groups alike. Societal indicators of reductions in human energy expenditure and hikes in SB during the past several decades are particularly striking. In recent decades, affluent populations have become increasingly sedentary, with many adults spending $70 \%$ or more of their waking hours sitting [25]. This risk of SB was reported in 2010, and in the results of the present study too, the rate of $\mathrm{SB}$ on the day off was comparable, and it was inferred that for nurses working a 16-hour shift in a two-shift system, it was the required activity level to recover from fatigue following the night shift. Therefore, it is not sufficient to merely increase the level of activity. It is equally important to propose ways to spend time in SB and activity content that leads to fatigue recovery.

We found that the physical activity intensity of nurses working a 16-hour shift in a two-shift system tended to be more commonly moderate or greater in non-pregnant women during the working hours of day shifts and night shifts. Furthermore, the feeling of fatigue could be reduced by spending time doing low intensity physical activity in pregnant and non-pregnant nurses alike on their day off following a night shift.

\section{Limitations}

The present study has two limitations. First, the details of activities in the physical activity intensities were not clarified. Second, in terms of background information on the four institutions where the subjects were employed, although the patient-nurse ratio was $7: 1$, the number of beds varied from 160 to 400 beds.

\section{Conclusions}

Our investigation revealed that both pregnant and non-pregnant nurses experienced increased fatigue before and after day shifts as well as night shifts but recovered by sleeping at night. Furthermore, it was found that indulging in physical activity of intensity comparable to SB on one's day off promoted fatigue recovery. We found that when compared to pregnant nurses, non-pregnant nurses experienced a persistent sympathetic nerve predominant stressful state from sleeping after the day shift through the night shift. 
Citation: Ooshige N, Yamaguchi T, Nakashima M, Kimura R, Tobina T, et al. (2021) A Comparison of Fatigue, Level of Physical Activity, and Sleep Quality in Pregnant and Non-Pregnant Nurses Working 16-Hour Night Shifts in Japan. Int J Nurs Clin Pract 8: 344. doi: https://doi.org/10.15344/2394-4978/2021/344

\section{Competing Interests}

The authors declares that they have no competing interests.

\section{Funding}

This research was supported by a Grant-In-Aid for Scientific Research from the Japan Society for Promotion of Science (No.19K10845)

\section{Author Contributions}

Study conceptualization and design: Narumi Ooshige, Tae Yamaguchi, Mitsuyo Nakashima, Ryohei Kimura, Takuro Tobina, Miyuki Nagamatsu, Yumiko Kuraoka.

Data collections: Narumi Ooshige, Tae Yamaguchi and Takuro Tobina

\section{Supervision: Narumi Ooshige}

Original draft: Narumi Ooshige, Ryohei Kimura and Takuro Tobina.

Critical revisions \& editing: Narumi Ooshige, Tae Yamaguchi, Mitsuyo Nakashima, Ryohei Kimura, Takuro Tobina, Miyuki Nagamatsu, Yumiko Kuraoka.

\section{References}

1. Vetter C, Devore EE, Wegrzyn LR, Massa J,Speizer FE, et al. (2016) Association between rotating night shift work and risk of coronary heart disease among women. JAMA 315: 1726-1734.

2. Min A, Min H, Hong HC (2019) Work schedule characteristics and fatigue among rotating shift nurses in hospital setting: An integrative review. J Nurs Manag 27: 884-895.

3. Japan federation of Medical Workers Union (2021) 2020ed night shift factfinding survey. Medical labor.

4. Ooshige N Yamaguchi T, Nakashima M Minematsu $\mathrm{K}$, Tsunawake $\mathrm{N}$, et al. (2018) Factors Influencing Cumulative Fatigue Symptoms in Female Nurses Working in Two Shifts Rotation System in Japan. International Journal of Nursing \& Clinical Practices 5: 270.

5. Ministry of Health, Labor and Welfare (2021) Current status and transition of nursing staff.

6. Sedov ID, Cameron EE, Madigan S, Tomfohr-Madsen LM (2018) Sleep quality during pregnancy: A meta-analysis. Sleep Med Rev 38: 168-176.

7. Watanabe A, Manabe E, Izumi M, Uematsu S, Tanaka H, et al. (2018) Sleep, anxiety and depression variations and their associated characteristics during pregnancy and to the postpartum period development. The Japanese Journal of Psychology 89: 130-138.

8. Yang Y, Shin JC, Li D, An R (2017) Sedentary Behavior and Sleep Problems: a Systematic Review and Meta-Analysis. Int J Behav Med 24: 481-492.

9. Ellingson LD, Kuffel AE, Vack NJ, Cook DB (2014) Active and sedentary behaviors influence feelings of energy and fatigue in women. Med Sci Sports Exerc 46: 192-200.

10. Tachi N (2002) The proposal and revised work progress of a new edition of "Jakaku-sho Shirabe". J Sci Labor 57: 299-304.

11. Doi Y, Minowa M, Okawa M, Uchiyama M (1998) Development of the Japanese version of the Pittsburgh Sleep Quality Index. Japanese Journal of Psychiatry Treatment 13: 755-763.

12. Takada H, Takada M, Kitayama A (2005) The Significance of LF-component and HF-component which Resulted from Frequency Analysis of Heart Rate and the Coefficient of the Heart Rate Variability-Evaluation of Autonomic Nerve Function by Acceleration Plethysmography. Health Evaluation and Promotion 32: 12-20.

13. Hayano J, Okada A, Yasuma F (1996) Biological Significance of Heart Rate Variability. Journal of Artificial Organs 25: 870-880.

14. Kosugo R, Fujii H (2002) Harmony of labor and health: CFSI manual. Institute for the Science of Labo, Kanagawa, Japan: Institute for Science of Labor.
15. Nakagawa C (2016) Measurement and Analyses of Bioelectric Phenomena and Others (5) Autonomic Indices. Measurement technique for Ergonomics 52: 6-12.

16. Shinozaki R, Matsui $T$ (2018) Application of the Biological Information Measured with a Small Heart Rate Sensor. Journal of Clinical Anesthesia 42: 879-886.

17. Kumahara H, Schutz $Y$, Ayabe M, Yoshioka M, Yoshitake $Y$, et al. (2004) The use of uniaxial accelerometry for the assessment of physical-activityrelated energy expenditure: a validation study against whole-body indirect calorimetry. Br J Nutr 91: 235-243.

18. HARVARD (2021) The Nutrition Source

19. Watanabe $M$, Akamatsu $Y$, Furui $H$, Tomita $T$, Watanabe $T$, et al. (2004) Effects of Changing Shift Schedules from a Full day to a Half-day Shift before a Night Shift on Physical Activities and Sleep Patterns of Single Nurses and Married Nurses with Children. Ind Health 42: 34-40.

20. Bacaro V, Benz F, Pappaccogli A, Bartolo PD, Johann AF, et al. (2019) Interventions for sleep problems during pregnancy: A systematic review. Sleep Med Rev 50: 101234.

21. Stawarczyk D, François C, Wertz J, D'Argembeau A (2020) Drowsiness or mind-wandering? Fluctuations in ocular parameters during attentional lapses. Biol Psychol 156: 107950.

22. Kosugo R (1991) Validity and Reliability of Cumulative Fatigue Symptoms Index. Journal of Science of Labor 67: 145-157.

23. Ichie K, Ohsawa I, Sato Y (2004) Cumulative Fatigue Symptoms among Caregivers in Japan. Environ Health Prev Med 9: 214-219.

24. Han K, Trinkoff AM (2014) Factors Associated with Work-Related Fatigue and Recovery in Hospital Nurses Working 12-Hour Shifts. Workplace Health Saf 62: 409-414.

25. Owen N, Sparling PB, Healy GN, Dunstan DW, Matthews CE, et al. (2010) Sedentary Behavior: Emerging Evidence for a New Health Risk. Mayo Clin Proc 85: 1138-1141. 